\title{
Effect of Horndeski theory on weak deflection angle using the Gauss-Bonnet theorem
}

\author{
Ali Övgün, ${ }^{1, *}$ Yashmitha Kumaran, ${ }^{1, \dagger}$ Wajiha Javed, ${ }^{2, \ddagger}$ and Jameela Abbas ${ }^{3, \S}$ \\ ${ }^{1}$ Physics Department, Eastern Mediterranean University, \\ Famagusta, 99628 North Cyprus via Mersin 10, Turkey. \\ ${ }^{2}$ Division of Science and Technology, University of Education, Township-Lahore, Pakistan \\ ${ }^{3}$ Department of Mathematics, University of Education, \\ Township, Lahore-54590, Pakistan.
}

(Dated: June 2, 2021)

\begin{abstract}
The main goal of this paper is to study the weak gravitational lensing by Horndeski black hole in weak field approximation. In order to do so, we exploit the Gibbons-Werner method to the optical geometry of Horndeski black hole and implement the Gauss-Bonnet theorem to accomplish the deflection angle of light in weak field region. Furthermore, we have endeavored to extend the scale of our work by comprising the impact of plasma medium on the deflection angle as properly. Later, the graphical influence of the deflection angle of photon on Horndeski black hole in plasma and nonplasma medium is examined.
\end{abstract}

PACS numbers: 95.30.Sf, 98.62.Sb, 97.60.Lf

Keywords: Weak gravitational lensing; Horndeski black hole; Deflection angle; Gauss-Bonnet theorem

\section{INTRODUCTION}

Gravitational physics has been an area of interest for centuries among physicists from diverse disciplines. The orbit of Mercury served as the foremost revelation of the requirement to refine Newtonian gravitational theory. Substantiating Lorentz Transformations and General Theory of Relativity encouraged many researchers to reform Newtonian gravity in an attempt to surpass it's limitations on high speeds, extreme gravity and particle nature. The most triumphant vindication to the Newtonian "missing mass" problem is Dark Matter hypothesis [1]. While this theory has numerous merits, there were disputes on introducing a new form of matter, suggesting the essential modification of Newtonian dynamics to account for the same. Hitherto, the idea of Modified Gravity was proposed, emerging as the widely accredited competitor of General Relativity. But, due to theoretical complexities and observational inadequacies, this approach is transcended by the Scalar-tensor Theory; it efficiently consolidates the scalar field (mass) with the metric (gravity) in the recent years [2-9].

Conversely, cluster of mass bending light to create a lensing effect - thus, acting as a magnifying glass and revealing inconspicuous details - validated the existence of dark matter [10-13]. This phenomena, known as gravitational lensing, is categorised into strong lensing, weak lensing and micro-lensing [14-59]. Whisker has used a strong field limit approach to investigate the gravitational lensing properties of braneworld black holes [34, 35]. Moreover, Keeton and Petters have provided a comprehensive analytical formalism for gravitational lensing due to a braneworld black hole described by the Garriga-Tanaka metric [36]. Bin-Nun has studied the properties of gravitational lensing by black holes in the Randall-Sundrum II braneworld [37]. Eiroa and Sendra have investigated weak and strong gravitational lensing by massless braneworld black holes [38].

In this paper, we are interested in weak lensing in which the distortions of the light source are high enough to be just detected, but too low to be able to promptly determine it's salient features. The typical mode of perpetuation is to calculate the angle of deflection of light, and herein, Gauss-Bonnet theorem is employed, mathematically expressed for a surface $S$ as: [60]

$$
\iint_{D} K d S+\int_{\partial D} \kappa d t+\sum_{i} \alpha_{i}=2 \pi \chi(D)
$$

where, $K$ is the Gaussian curvature, $\kappa$ is the geodesic curvature, $\alpha_{i}$ is the exterior angle with $i^{\text {th }}$ vertex, and $\chi$ is the Euler characteristic of topology, altogether defined with $g$ as the Riemannian metric of the manifold of lens in

\footnotetext{
*Electronic address: ali.ovgun@emu.edu.tr

${ }^{\dagger}$ Electronic address: y.kumaran13@gmail.com

‡Electronic address: wajiha.javed@ue.edu.pk; wajihajaved84@yahoo.com

$\S$ Electronic address: jameelaabbas30@gmail.com
} 
a surface domain $(D, \chi, g)$ outside the light trajectory. Some other works can be found in Refs. [61-104]. For an asymptotically flat line-of-sight, the deflection angle, is given by: [60]

$$
\hat{\alpha}=-\iint_{D} K d S
$$

With this method as the basis, consider a black hole that obeys the most generalised Scalar-tensor theory in fourdimensional spacetime which yields second-order equations of motion: the Horndeski theory - it characterizes the scalar field as a new degree of freedom, evolving into a Lagrangian that unravels second-order field equations of motion. The action is written as: $[105,106]$

$$
S=\int d^{4} x \sqrt{-g} \mathcal{L}
$$

with the generalised Galilean Lagrangean, $\mathcal{L} \equiv\left(\mathcal{L}_{2}+\mathcal{L}_{3}+\mathcal{L}_{4}+\mathcal{L}_{5}\right)$ expanded such that:

$$
\begin{aligned}
& \mathcal{L}_{2}=G_{2} \\
& \mathcal{L}_{3}=-G_{3} \square \phi \\
& \mathcal{L}_{4}=G_{4} R+G_{4 X}\left[(\square \phi)^{2}-\left(\nabla_{\mu} \nabla_{\nu} \phi\right)^{2}\right] \\
& \mathcal{L}_{5}=G_{5} G_{\mu \nu} \nabla^{\mu} \nabla^{\nu} \phi-\frac{G_{5 X}}{6}\left[(\square \phi)^{3}-3 \square \phi\left(\nabla_{\mu} \nabla_{\nu} \phi\right)^{2}+2\left(\nabla_{\mu} \nabla_{\nu} \phi\right)^{3}\right]
\end{aligned}
$$

It is to be noted that $G_{i}$ is an arbitrary function of scalar field, $\phi$ and kinetic energy, $X \equiv-\partial_{\mu} \phi \partial^{\mu} \phi / 2$ that depends on the curvature and the coupling of the scalar field, whereas, the subscript $X$ denotes the derivative with respect to $X$. Also, $g_{\mu \nu}$ is the metric tensor with a determinant $g, R$ is the Ricci scalar, $G_{\mu \nu}$ is the Einstein tensor, along with:

$$
\square \phi \equiv g^{\mu \nu} \nabla_{\mu} \nabla_{\nu} \phi \quad ; \quad\left(\nabla_{\mu} \nabla_{\nu} \phi\right)^{2} \equiv \nabla_{\mu} \nabla_{\nu} \phi \nabla^{\mu} \nabla^{\nu} \phi \quad ; \quad\left(\nabla_{\mu} \nabla_{\nu} \phi\right)^{3} \equiv \nabla_{\mu} \nabla_{\nu} \phi \nabla^{\nu} \nabla^{\sigma} \phi \nabla_{\sigma} \nabla_{\mu} \phi
$$

Recent works in multi-messenger astronomy of detecting gravitational waves from a binary neutron star merger (GW170817) [107] have imposed a few constraints on the scalar-tensor theories. Since the recorded times of arrival of the gravitational waves and the electromagnetic waves vary under a minute, gravitational waves with an electromagnetic counterpart measured from a cosmological event might culminate the idea of cosmic acceleration as a consequence of modifying scalar-tensor gravity for the Horndeski theory. Coupling the scalar field to curvature is observed to impact the speed of gravitational waves. In order to be consistent with these observations, the cosmological solution and the equivalence principle the quintic and quartic models are neglected restricting our calculations to linear observables [108].

Building on this premise, this paper is organized as follows: In section 2, we review some basic concepts about Horndeski black hole and we evaluate the Gaussian optical curvature for deflection angle and compute the deflection angle by applying GBT, then we discuss the plots in 3. In section 4, the deflection angle of light for Horndeski black hole in a plasma medium is obtained and discuss the plots in $\mathbf{5}$. The last section comprises of concluding remarks.

\section{EVALUATION OF PHOTON LENSING FOR HORNDESKI BLACK HOLES}

We consider the particular case of the action in which $G_{2}=\eta X, G_{4}=\zeta+\beta \sqrt{-X}$ and $G_{3}=G_{5}=0$, where $\eta$ and $\beta$ are dimensionless parameters and $\zeta=M_{p l}^{2} /(16 \pi)$. Then, the action takes the explicit form

$$
S=\int \sqrt{-g} d^{4} r\left\{\left[\zeta+\beta \sqrt{\frac{\partial \phi)^{2}}{2}}\right] R-\frac{\eta}{2}(\partial \phi)^{2}-\frac{\beta}{\sqrt{2(\partial \phi)^{2}}}\left[(\phi)^{2}-\left(\nabla_{\mu} \nabla_{\nu} \phi\right)^{2}\right]\right\} .
$$

The coefficient $\zeta$ gives the Einstein-Hilbert part of the action; one of the parameters $\eta$ and $\beta$ can be absorbed into the scalar field by means of a redefinition, but we will not do it in order to trace the origin of the different terms. The field equations resulting from equation (9) admit a static, spherically symmetric, and asymptotically flat solution of the form $[105,106]$

$$
d s^{2}=-A(r) d t^{2}+B(r) d r^{2}+C(r)\left(d \theta^{2}+\sin ^{2} \theta d \phi^{2}\right)
$$


With

$$
A(r)=1-\frac{\mu}{r}-\frac{\beta^{2}}{2 \zeta \eta r^{2}}, B(r)=\frac{1}{A(r)}, C(r)=r^{2}
$$

Note that this solution looks like black hole in braneworld gravity [34]. The integration constant $\mu$ can be interpreted as twice the black hole mass, i.e $\mu=2 M$. The parameters $\beta$ and $\eta$ should share the same sign and scalar field is given by

$$
\begin{gathered}
\phi(r)= \pm 2 \sqrt{\frac{\zeta}{\eta}}\left\{\arctan \left[\frac{\beta^{2}+\zeta \eta \mu r}{\beta \sqrt{2 \zeta \eta r(r-\mu)-\beta^{2}}}\right]-\arctan \left(\frac{\mu}{\beta} \sqrt{\frac{\zeta \eta}{2}}\right)\right\}, \quad \text { if } \beta>0 \text { and } \eta>0 \\
\phi(r)= \pm 2 \sqrt{\frac{\zeta}{-\eta}}\left\{\arctan \left[\frac{\beta^{2}+\zeta \eta \mu r}{\beta^{2}-\beta \sqrt{2 \zeta \eta r(r-\mu)}}\right]+\arctan \left(\frac{\mu}{\beta} \sqrt{\frac{-\zeta \eta}{2}}\right)\right\}, \quad \text { if } \beta<0 \text { and } \eta<0
\end{gathered}
$$

The geometry is singular as the origin of coordinates. It is useful to define the parameter $\gamma=\beta^{2} /(2 \zeta \eta)$ in order to simplify the notation. We can see that a negative value of $\gamma$ makes this metric identical to the Reissner-Nordstrom metric, with the squared charge given by $Q^{2}=-\gamma$.

Now using the equation (12), we write the null geodesics $\left(d s^{2}=0\right)$, the black hole optical spacetime can be simply written in equatorial plane $\theta=\frac{\pi}{2}$ :

$$
d t^{2}=\frac{B(r)}{A(r)} d r^{2}+\frac{C(r)}{A(r)} d \phi^{2} .
$$

The Gaussian curvature that is proportional to the Ricci scalar can be defined as:

$$
\mathcal{K}=\frac{R_{\text {icciScalar }}}{2}
$$

using the non-zero christopher symbol the Gaussian optical curvature for Horndeski black hole can be computed as

$$
\mathcal{K}=-3 \frac{\tilde{\gamma}}{r^{4}}+\left(-r^{-3}+3 \frac{\tilde{\gamma}}{r^{5}}\right) \mu+\mathcal{O}\left(\mu^{2}, \tilde{\gamma}^{2}\right) .
$$

Let us bear in mind the GBT for a two dimensional manifold. Regarding to this, we take into account a regular domain $M_{R}$ arranged by 2-dimensional surface $S$ with Riemannian metric $\hat{g}_{i j}$, in conjunction with boundary $\partial M_{R}=$ $\gamma_{g} \cup C_{R}$, so GBT allows a coupling between the geometry and topology in terms of the following relation

$$
\iint_{M_{R}} \mathcal{K} d S+\oint_{\partial M_{R}} k d \sigma+\sum_{j} \hat{\theta}_{j}=2 \pi \mathcal{X}\left(M_{R}\right)
$$

Here $\mathcal{K}$ represented as the Gaussian optical curvature. It is a well-known fact that for regular domain the Euler characteristic $\mathcal{X}_{M_{R}}=1$, while $k$ is known as a geodesic curvature and is defined as [109]

$$
k=g^{o p}\left(\nabla_{\dot{\gamma}} \dot{\gamma}, \ddot{\gamma}\right)
$$

having the unit speed condition $g^{o p}(\dot{\gamma}, \dot{\gamma})=1$, where $\ddot{\gamma}$ is the unit acceleration vector. In the case of $R \rightarrow \infty$, the corresponding jump angles are taken as $\pi / 2$ (in short $\theta_{O}+\theta_{S} \rightarrow \pi$ ). using the fact that, from geodesic there is a zero contribution i.e. $k\left(\gamma_{\tilde{g}}\right)=0$, we shall pursue a contribution by virtue of the curve $C_{R}$ and this contribution can be computed as

$$
k\left(C_{R}\right)=\left|\nabla_{\dot{C}_{R}} \dot{C}_{R}\right|
$$

Let us consider $C_{R}:=r(\phi)=R=$ const, while $R$ endows the distance from the coordinate origin. The radial component of the geodesic curvature states as

$$
\left(\nabla_{\dot{C}_{R}} \dot{C}_{R}\right)^{r}=\dot{C}_{R}^{\phi}\left(\partial_{\phi} \dot{C}_{R}^{\phi}\right)+\Gamma_{\phi \phi}^{r}\left(\dot{C}_{R}^{\phi}\right)^{2} .
$$


In the above equation, we note that the first term vanishes. Hence the second term can be calculated using the unit speed condition. Then, $\kappa$ is calculated as:

$$
\lim _{R \rightarrow \infty} \kappa\left(C_{R}\right)=\lim _{R \rightarrow \infty}\left|\nabla_{\dot{C}_{R}} \dot{C}_{R}\right| \rightarrow \frac{1}{R}
$$

We take the large limits of the radial distance, and find:

$$
\lim _{R \rightarrow \infty} \mathrm{d} t \rightarrow R \mathrm{~d} \phi
$$

Considering the above conditions with $\kappa\left(C_{R}\right) \mathrm{d} t=\mathrm{d} \phi$ and using the straight line approximation $r=b / \sin \phi$, the form invariant deflection angle follows as:

$$
\Theta=-\int_{0}^{\pi} \int_{b / \sin \phi}^{\infty} \mathcal{K} d S
$$

This equation inscribe the global impact on the lensing of particles on account of the fact that one has to integrate over the optical domain of integration outside the enclosed mass where $d S=\sqrt{\operatorname{det} g} d r d \phi$.

Using equation (17) into the above equation, we find the deflection angle for Horndeski black hole in weak field limits to be:

$$
\Theta=\frac{2 \mu}{b}+\frac{3 \pi \tilde{\gamma}}{4 b^{2}}+\mathcal{O}\left(\mu^{2}, b^{3}\right)
$$

\section{Graphical Analysis of Weak deflection angle}

This segment is devoted to scrutinize the graphical effect of deflection angle $\Theta$ on Horndeski black holes. Furthermore, describe the physical eminence of these plots by examining the stable and unstable state of black hole to analyze the impact of $\tilde{\gamma}, \mu$ and impact parameter $b$ on deflection angle. The following provides the analysis of deflection angle $\Theta$ with impact parameter $b$ for different values of $\tilde{\gamma}$ and $\mu$.

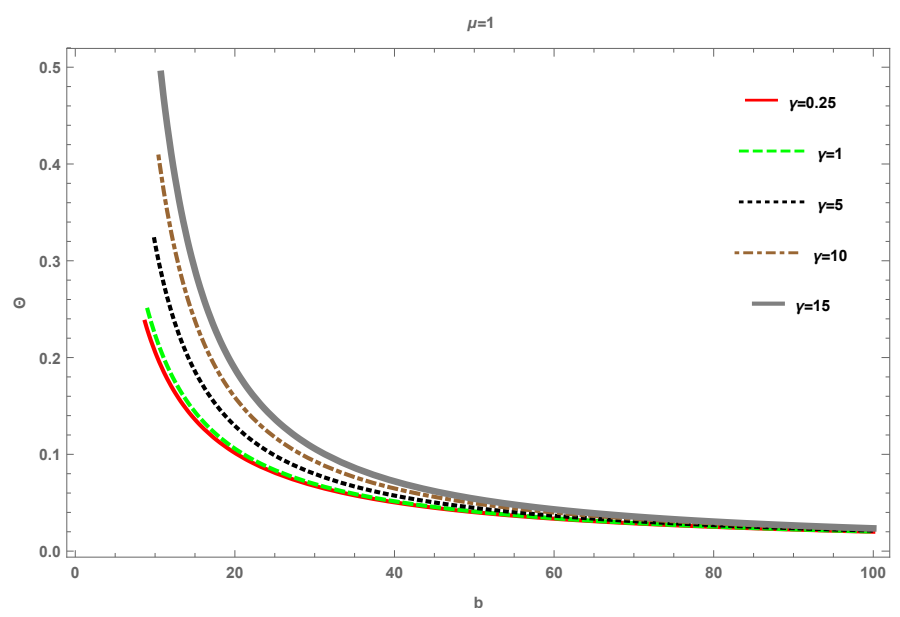

Figure 1: $\Theta$ versus $b$ to see the influence of $\tilde{\gamma}$ parameter on weak deflection angle.

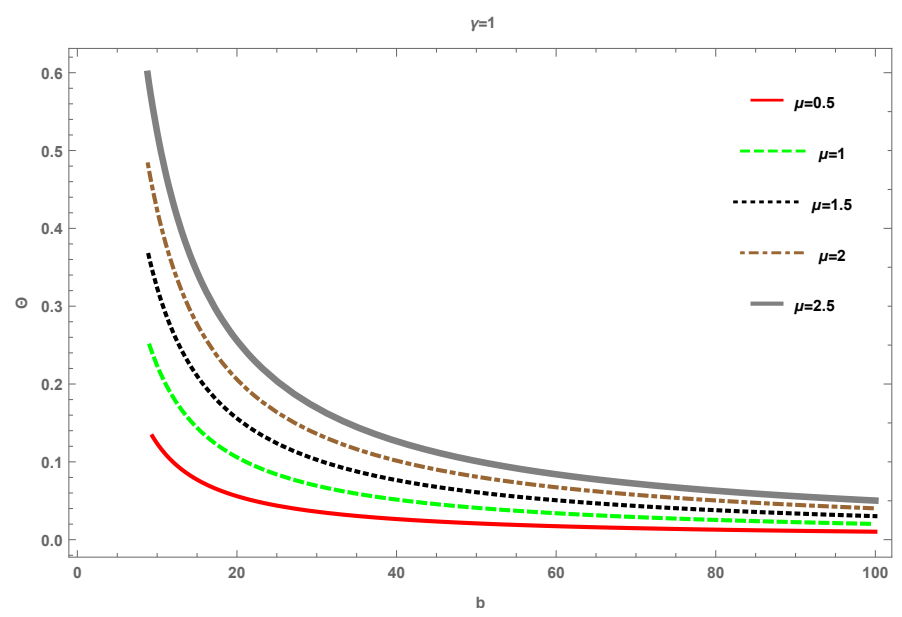

Figure 2: $\Theta$ versus $b$ to see the influence of $\mu$ parameter on weak deflection angle.

- Figures 1 and 2 exhibit the effect of $\Theta$ versus $b$ for different parameters. The deflection angle is analyzed to have an indirect relation with the impact parameter; one can choose $b \in[1,50]$ to sustain stable behavior.

1. Figure 1 shows the graphical influence of deflection angle against $b$ with varying $\tilde{\gamma}$ and fixed $\mu$. For this, we observe that negative $\tilde{\gamma}$ makes this metric identical to RN solution. It is further investigated if the deflection angle increases exponentially when $\tilde{\gamma}$ increases.

2. Figure 2 depicts that the deflection angle gradually increase by increasing the mass term $\mu$. 
In figure 1 , the parameter $\tilde{\gamma}$ is seen to affect the deflection angle more for lower values of the impact parameter. This susceptibility could be utilized for various computations involving the effects of $b$ on $\Theta$ for a changing $\tilde{\gamma}$. Also, it can be noticed that figure 2 varies more abruptly with an increasing $\mu$ compared to figure 1 with an increasing $\tilde{\gamma}$. This suggests that although the mass term dominates, the influence of the parameter $\tilde{\gamma}$, however small, is not negligible.

\section{WEAK DEFLECTION ANGLE IN A PLASMA MEDIUM}

With the objective to mainstream the impact of plasma, keep in mind the case whilst light travels from vacuum to a hot, ionized gas medium. Consider $v$ be the velocity of photon within plasma. The refractive index, $n(r)$ is defined by: [80]

$$
n(r) \equiv \frac{c}{v}=\frac{1}{d r / d t} \quad(c=1)
$$

The equation for refractive index by Horndeski black hole is computed as [80],

$$
n(r)=\sqrt{1-\frac{\omega_{e}^{2}}{\omega_{\infty}^{2}} A(r)},
$$

here $\omega_{e}$ referred to as electron plasma frequency and $\omega_{\infty}$ introduced as photon frequency found by an observer at infinity. Therefore, the equivalent optical metric designated as:

$$
d \sigma^{2}=g_{j k}^{o p t} d x^{j} d x^{k}=\frac{n^{2}(r)}{A(r)}\left[B(r) d r^{2}+C(r) d \phi^{2}\right] .
$$

The optical Gaussian curvature for the above optical metric is calculated as follows

$$
\mathcal{K}=\left(-r^{-3}-3 / 2 \frac{\omega_{e}^{2}}{\omega_{\infty}^{2} r^{3}}\right) \mu+\left(-3 r^{-4}-5 \frac{\omega_{e}^{2}}{\omega_{\infty}^{2} r^{4}}+\left(3 r^{-5}+13 \frac{\omega_{e}^{2}}{\omega_{\infty}^{2} r^{5}}\right) \mu\right) \tilde{\gamma}
$$

Hence, we get also that:

$$
\left.\lim _{r \rightarrow \infty} k_{g} \frac{d \tilde{\sigma}}{d \phi}\right|_{C_{R}}=1
$$

We use straight line approximation $r=b / \sin \phi$, for the limit $r \rightarrow \infty$, then GBT stated as [80]

$$
\left.\lim _{x \rightarrow \infty} \int_{0}^{\pi+\Theta}\left[k_{g} \frac{d \tilde{\sigma}}{d \phi}\right]\right|_{C_{R}} d \phi=\pi-\lim _{x \rightarrow \infty} \int_{0}^{\pi} \int_{b / \sin \phi}^{x} \mathcal{K} d S .
$$

After simplification, we obtain

$$
\Theta \simeq \frac{2 \mu}{b}+\frac{3 \pi \tilde{\gamma}}{4 b^{2}}+\frac{3 \mu}{b} \frac{\omega_{e}^{2}}{\omega_{\infty}^{2}}+\frac{5 \pi \tilde{\gamma}}{4 b^{2}} \frac{\omega_{e}^{2}}{\omega_{\infty}^{2}}
$$

The above results shows that the photon rays are moving in a medium of homogeneous plasma. The plasma effect can be removed by neglecting $\omega_{e} / \omega_{\infty}$.

\section{Graphical Analysis of Weak deflection angle in a plasma medium}

This subsection is devoted to study the graphical effect of deflection angle $\Theta$ on Horndeski black holes. In addition, we exemplify the physical impact of these graphs to review the effect of plasma term $\omega_{e} / \omega_{\infty}, \tilde{\gamma}$ and impact parameter $b$ on deflection angle. The deflection angle $\Theta$ is analyzed with respect to the impact parameter $b$ in a plasma medium. For this, we fixed $\mu=2, \pi=3.14$ and for the sake of simplicity take $\omega_{e} / \omega_{\infty}=P$. 


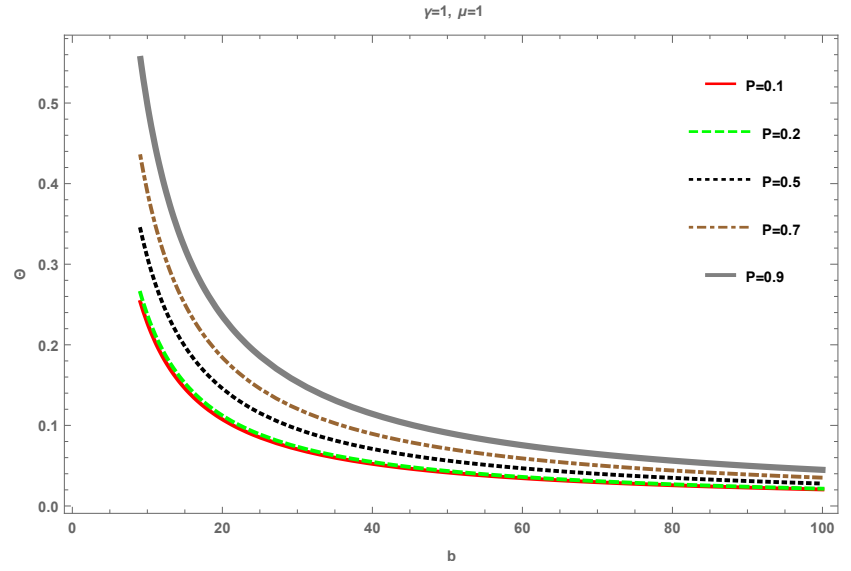

Figure 3: Figure reveals the impact of $\Theta$ over $b$ for different ranges of $P$ with fixed $\tilde{\gamma}$ and and fixed $\mu$.

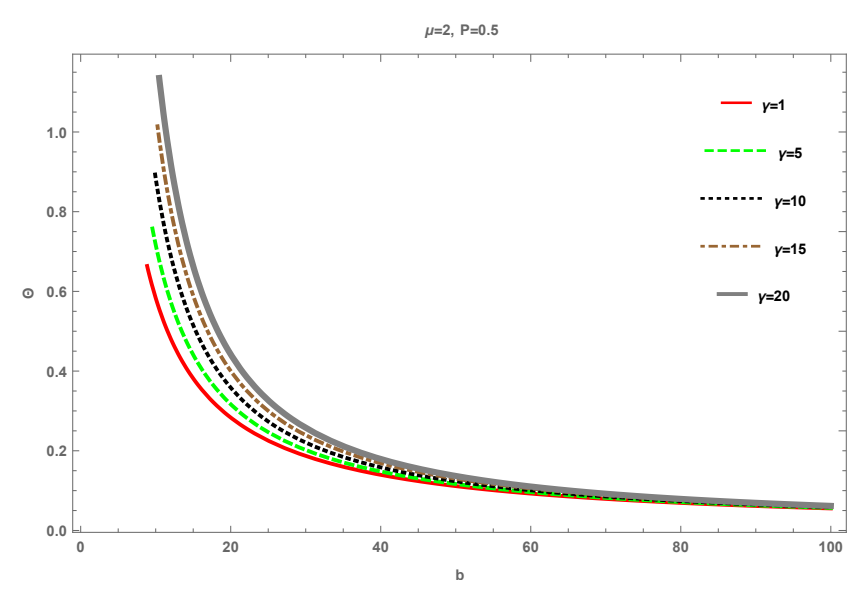

Figure 4: Figure reveals the impact of $\Theta$ over $b$ for different ranges of $\tilde{\gamma}$ with fixed $P$ and fixed $\mu$.

- Figures 3 and 4 demonstrate the effects of varying parameters on the deflection angle with respect to the impact parameter graphically.

1. Figure 3 interprets the significance of varying $P$ where the positive behavior of $\Theta$ is analyzed only for the range $0.1 \leq P \leq 0.9$. Increasing the value of $P$ (for plasma medium) increases the weak deflection angle.

2. Figure 4 illustrates an exponential increase in the deflection angle for an increasing $\tilde{\gamma}$.

The nature of these graphs is similar to the plots corresponding to non-plasma medium. Figure 3 indicates the nontrivial consequences of an increasing $P$ : as $P$ approaches 1 , the influence on $\Theta$ is seen to increase drastically. Figure 4 depicts the behaviour of the deflection angle against the impact parameter in the presence of plasma, which is doubled when compared to figure 1. It is apparent that the deflection angle is rather sensitive to plasma especially for low $b$.

\section{CONCLUSION}

In this paper, we have examined the deflection angle of photon by Horndeski black hole in weak field limits. We found the corresponding optical geometry of a Horndeski black hole followed by employing the straight line approximation as dictated by the Gauss-Bonnet theorem and estimating the deflection angle in leading order terms. The acquired deflection angle is measured by integrating a domain outside the impact parameter, which shows the global influence of gravitational lensing. Moreover, we have computed the deflection angle of photon by Horndeski black hole in plasma medium. It is analyzed that the acquired deflection angle is greater by increasing the plasma medium parameter; similarly, the term $\tilde{\gamma}$ increases the weak deflection angle as well. Note that the deflection angle for Horndeski black hole reduces to the deflection angle for Schwarzschild black hole when $\mu=2 M$ at the first-order term of impact parameter. Moreover, for the case of $\gamma=-Q^{2}$, the result becomes the deflection angle for ReissnerNordstrom black hole at the first-order term of impact parameter. The charge makes a small correction in the second order term which causes the weak deflection angle to be smaller than that of the Schwarzschild case, agreeing with $[110,111]$.

In conclusion, we have inspected the influence on the deflection angle of a Horndeski black hole graphically for non-plasma as well as plasma medium. It is evident that the deflection angle increases when the photon rays flow through a medium of homogeneous plasma.

[1] R. H. Sanders and S. S. McGaugh, Ann. Rev. Astron. Astrophys. 40, 263 (2002).

[2] S. X. Tian and Z. H. Zhu, Phys. Rev. D 100, no. 12, 124059 (2019).

[3] S. Nojiri and S. D. Odintsov, Phys. Lett. B 631, 1 (2005).

[4] E. Berti et al., Class. Quant. Grav. 32, 243001 (2015).

[5] S. Nojiri, S. D. Odintsov and V. K. Oikonomou, Phys. Rept. 692, 1 (2017). 
[6] S. Capozziello and M. De Laurentis, Phys. Rept. 509, 167 (2011).

[7] G. W. Horndeski, Int. J. Theor. Phys. 10, 363 (1974).

[8] D. Blas, O. Pujolas and S. Sibiryakov, Phys. Rev. Lett. 104, 181302 (2010).

[9] J. Sakstein and B. Jain, Phys. Rev. Lett. 119, no. 25, 251303 (2017).

[10] R. A. Flores and J. R. Primack, Astrophys. J. 427, L1 (1994)

[11] B. Moore, S. Ghigna, F. Governato, G. Lake, T. R. Quinn, J. Stadel and P. Tozzi, Astrophys. J. 524, L19 (1999)

[12] D. M. Wittman, J. A. Tyson, D. Kirkman, I. Dell'Antonio and G. Bernstein, Nature 405, 143 (2000)

[13] D. Clowe, M. Bradac, A. H. Gonzalez, M. Markevitch, S. W. Randall, C. Jones and D. Zaritsky, Astrophys. J. 648, L109 (2006)

[14] M. Bartelmann and P. Schneider, Phys. Rept. 340, 291 (2001)

[15] N. Kaiser, G. Squires and T. J. Broadhurst, Astrophys. J. 449, 460 (1995)

[16] K. S. Virbhadra and G. F. R. Ellis, Phys. Rev. D 62, 084003 (2000)

[17] V. Bozza, Phys. Rev. D 66, 103001 (2002)

[18] K. S. Virbhadra and G. F. R. Ellis, Phys. Rev. D 65, 103004 (2002).

[19] H. Hoekstra and B. Jain, Ann. Rev. Nucl. Part. Sci. 58, 99 (2008)

[20] C. Y. Peng, C. D. Impey, H. W. Rix, C. S. Kochanek, C. R. Keeton, E. E. Falco, J. Lehar and B. A. McLeod, Astrophys. J. 649, $616(2006)$

[21] K. S. Virbhadra and C. R. Keeton, Phys. Rev. D 77, 124014 (2008)

[22] C. R. Keeton, astro-ph/0102340.

[23] C. R. Keeton and A. O. Petters, Phys. Rev. D 72, 104006 (2005)

[24] A. Bhadra, Phys. Rev. D 67, 103009 (2003)

[25] G. N. Gyulchev and S. S. Yazadjiev, Phys. Rev. D 75, 023006 (2007)

[26] G. W. Gibbons, C. M. Warnick and M. C. Werner, Class. Quant. Grav. 25, 245009 (2008)

[27] G. W. Gibbons and C. M. Warnick, Phys. Rev. D 79, 064031 (2009)

[28] D. C. Latimer, Phys. Rev. D 88, 063517 (2013)

[29] T. Elghozi, N. E. Mavromatos and M. Sakellariadou, Eur. Phys. J. C 77, no. 7, 445 (2017)

[30] W. G. Cao and Y. Xie, Eur. Phys. J. C 78, no. 3, 191 (2018).

[31] Y. K. Lim and Q. h. Wang, Phys. Rev. D 95, no. 2, 024004 (2017)

[32] J. Sultana, JCAP 1304, 048 (2013).

[33] P. Fleury, J. Larena and J. P. Uzan, Phys. Rev. Lett. 119, no. 19, 191101 (2017)

[34] R. Whisker, Phys. Rev. D 71, 064004 (2005)

[35] N. Dadhich, R. Maartens, P. Papadopoulos and V. Rezania, Phys. Lett. B 487, 1-6 (2000)

[36] C. R. Keeton and A. O. Petters, Phys. Rev. D 73, 104032 (2006)

[37] A. Y. Bin-Nun, Phys. Rev. D 81, 123011 (2010)

[38] E. F. Eiroa and C. M. Sendra, Phys. Rev. D 86, 083009 (2012)

[39] E. F. Eiroa, Braz. J. Phys. 35, 1113-1116 (2005)

[40] S. b. Chen and J. 1. Jing, Phys. Rev. D 80, 024036 (2009)

[41] E. F. Eiroa, G. E. Romero and D. F. Torres, Phys. Rev. D 66, 024010 (2002)

[42] C. Y. Wang, Y. F. Shen and Y. Xie, JCAP 1904, 022 (2019)

[43] S. Mao and B. Paczynski, Astrophys. J. 374, L37 (1991).

[44] M. Sharif and S. Iftikhar, Astrophys. Space Sci. 357, no. 1, 85 (2015).

[45] O. Kasikci and C. Deliduman, Phys. Rev. D 100, no. 2, 024019 (2019)

[46] R. Zhang and J. Jing, Eur. Phys. J. C 78, no. 10, 796 (2018).

[47] H. Arakida and M. Kasai, Phys. Rev. D 85, 023006 (2012)

[48] G. S. Bisnovatyi-Kogan and O. Y. Tsupko, Universe 3, no. 3, 57 (2017)

[49] C. Q. Liu, C. K. Ding and J. L. Jing, Chin. Phys. Lett. 34, no. 9, 090401 (2017)

[50] G. S. Bisnovatyi-Kogan and O. Y. Tsupko, Plasma Phys. Rep. 41, 562 (2015)

[51] B. Ahmedov, B. Turimov, Z. Stuchlík and A. Tursunov, Int. J. Mod. Phys. Conf. Ser. 49, 1960018 (2019).

[52] B. Turimov, B. Ahmedov, A. Abdujabbarov and C. Bambi, Int. J. Mod. Phys. D 28, no.16, 2040013 (2019).

[53] A. Abdujabbarov, B. Ahmedov, N. Dadhich and F. Atamurotov, Phys. Rev. D 96, no. 8, 084017 (2017).

[54] J. Schee, Z. Stuchlík, B. Ahmedov, A. Abdujabbarov and B. Toshmatov, Int. J. Mod. Phys. D 26, no. 5, 1741011 (2017).

[55] H. Ghaffarnejad, M. Amirmojahedi and H. Niad, Adv. High Energy Phys. 2018, 3067272 (2018)

[56] A. B. Aazami, C. R. Keeton and A. O. Petters, J. Math. Phys. 52, 102501 (2011)

[57] C. R. Keeton and A. O. Petters, Phys. Rev. D 73, 044024 (2006)

[58] Z. Horvath, L. A. Gergely, D. Hobill, S. Capozziello and M. De Laurentis, Phys. Rev. D 88, no.6, 063009 (2013)

[59] S. Capozziello, V. F. Cardone and A. Troisi, Phys. Rev. D 73, 104019 (2006)

[60] G. W. Gibbons and M. C. Werner, Class. Quant. Grav. 25, 235009 (2008).

[61] M. C. Werner, Gen. Rel. Grav. 44, 3047 (2012).

[62] A. Ishihara, Y. Suzuki, T. Ono, T. Kitamura and H. Asada, Phys. Rev. D 94, no. 8, 084015 (2016).

[63] I. Sakalli and A. Ovgun, EPL 118, no. 6, 60006 (2017).

[64] K. Jusufi, M. C. Werner, A. Banerjee and A. Övgün, Phys. Rev. D 95, no. 10, 104012 (2017).

[65] T. Ono, A. Ishihara and H. Asada, Phys. Rev. D 96, no. 10, 104037 (2017).

[66] K. Jusufi, I. Sakalli and A. Övgün, Phys. Rev. D 96, no. 2, 024040 (2017).

[67] A. Ishihara, Y. Suzuki, T. Ono and H. Asada, Phys. Rev. D 95, no. 4, 044017 (2017).

[68] K. Jusufi, A. Övgün and A. Banerjee, Phys. Rev. D 96, no. 8, 084036 (2017). 
[69] H. Arakida, Gen. Rel. Grav. 50, no. 5, 48 (2018).

[70] K. Jusufi and A. Övgün, Phys. Rev. D 97, no. 2, 024042 (2018).

[71] A. Övgün, I. Sakalli and J. Saavedra, JCAP 1810, no. 10, 041 (2018)

[72] A. Övgün, I. Sakalli and J. Saavedra, Annals Phys. 411, 167978 (2019).

[73] A. Övgün, G. Gyulchev and K. Jusufi, Annals Phys. 406, 152 (2019).

[74] K. Jusufi, A. Övgün, J. Saavedra, P. A. Gonzalez and Y. Vasquez, Phys. Rev. D 97, 124024 (2018).

[75] A. Övgün, K. Jusufi and I. Sakalli, Annals Phys. 399, 193 (2018).

[76] K. Jusufi and A. Övgün, Phys. Rev. D 97, no. 6, 064030 (2018).

[77] A. Övgün, Phys. Rev. D 98, no. 4, 044033 (2018)

[78] T. Ono, A. Ishihara and H. Asada, Phys. Rev. D 98, no. 4, 044047 (2018)

[79] T. Ono, A. Ishihara and H. Asada, Phys. Rev. D 99, no. 12, 124030 (2019).

[80] G. Crisnejo and E. Gallo, Phys. Rev. D 97, 124016 (2018).

[81] G. Crisnejo, E. Gallo and A. Rogers, Phys. Rev. D 99, no. 12, 124001 (2019).

[82] Z. Li and A. Övgün, Phys. Rev. D 101, no. 2, 024040 (2020).

[83] Z. Li and T. Zhou, Phys. Rev. D 101, no. 4, 044043 (2020).

[84] Z. Li and J. Jia, Eur. Phys. J. C 80, no. 2, 157 (2020).

[85] Z. Li, G. He and T. Zhou, Phys. Rev. D 101, no. 4, 044001 (2020).

[86] K. Jusufi, A. Övgün, A. Banerjee and I. Sakalli, Eur. Phys. J. Plus 134, no. 9, 428 (2019).

[87] A. Övgün, Universe 5, no. 5, 115 (2019).

[88] W. Javed, R. Babar, A. Ovgun, Phys. Rev. D 99, no. 8, 084012 (2019).

[89] W. Javed, A. Hazma and A. Övgün, Phys. Rev. D 101, no.10, 103521 (2020).

[90] W. Javed, J. Abbas, and A. Övgün, Eur. Phys. J. C 79, no. 8, 694 (2019).

[91] A. Övgün, Phys. Rev. D 99, no. 10, 104075 (2019).

[92] W. Javed, j. Abbas and A. Övgün, Phys. Rev. D 100, no. 4, 044052 (2019).

[93] K. Jusufi and A. Övgün, Int. J. Geom. Meth. Mod. Phys. 16, no. 08, 1950116 (2019).

[94] G. Crisnejo, E. Gallo, and J. R. Villanueva, Phys. Rev. D 100, no. 4, 044006 (2019).

[95] W. Javed, R. Babar, and A. Övgün, Phys. Rev. D 100, no. 10, 104032 (2019)

[96] K. de Leon and I. Vega, Phys. Rev. D 99, no. 12, 124007 (2019)

[97] Y. Kumaran and A. Övgün, Chin. Phys. C 44, no. 2, 025101 (2020).

[98] W. Javed, J. Abbas, Y. Kumaran and A. Övgün, Int. J. Geom. Meth. Mod. Phys. 18, no.01, 2150003 (2020).

[99] A. Övgün, I. Sakalli and J. Saavedra, Chin. Phys. C 44, no.12, 125105 (2020).

[100] A. Övgün, Turk. J. Phys. 44, no.5, 465-471 (2020)

[101] W. Javed, A. Hamza and A. Övgün, Mod. Phys. Lett. A 35, no.39, 39 (2020)

[102] W. Javed, M. B. Khadim and A. Övgün, Eur. Phys. J. Plus 135, no.7, 595 (2020)

[103] A. Övgün and İ. Sakallı, Class. Quant. Grav. 37, no.22, 225003 (2020)

[104] Z. Li and A. Övgün, Phys. Rev. D 101, no.2, 024040 (2020),

[105] E. Babichev, C. Charmousis and A. Lehébel, JCAP 1704, no. 04, 027 (2017).

[106] J. Badia and E. F. Eiroa, Eur. Phys. J. C 77, no. 11, 779 (2017).

[107] B. P. Abbott et al. [LIGO Scientific and Virgo], Phys. Rev. Lett. 119, no.16, 161101 (2017)

[108] T. Kobayashi, Rept. Prog. Phys. 82, no.8, 086901 (2019)

[109] G.W. Gibbons and M. C.Werner, Class. Quant. Grav. 25, (2008)235009.

[110] M. Sereno, Phys. Rev. D 67, 064007 (2003)

[111] S. Fernando and S. Roberts, Gen. Rel. Grav. 34, 1221-1230 (2002) 